\title{
Nochmals zur Wirkung des Asparagins auf den Stickstoffumsatz im Tierkörper.
}

\author{
Von
}

\section{Lehmanu.}

In Band 113 dieses Archivs S. 480 u. f. hat O. Kellner meine Mitteilung über die Wirkung des Asparagins usw. in Band 112 S. 339 u. f. einer Kritik unterzogen, die ich nicht ohne Widerspruch lassen kann.

Kellner hat die mitgeteilten Versuche aufgefasst, als wenn ihr Zweck wäre, in erster Linie die Fleischproduktion nach Verabfolgung einer Nährstoffmischung festzustellen. Das war aber, wie nicht nur aus dem Titel der Arbeit hervorgeht, durchaus nicht der Fall. Die Versuche sollten eben die Wirkung auf den Stickst offumsatz feststellen und ergaben das unbezweifelbare Resultat, dass nach gleichmässig steigenden Zulagen des Asparagins in Hüllen der Stickstoffumsatz erheblich weniger vergrössert, die Stickstoffausscheidung sehr viel träger den Zulagen folgen lässt als das freie Asparagin, - dass ferner die Steigerung des Stickstoffumsatzes durch verdautes Blutalbumin auf die Einheit Stickstoff annähernd die gleiche war wie bei dem eingehülsten Asparagin.

Eine eigentliche Stickstoffbilanz, welches Wort in der ganzen Arbeit nicht vorkommt, war gar nicht beabsichtigt aufzustellen. Soll dies geschehen, so müsste selbstverständlich die sogenannte Nachwirkung der N-Zulagen in Rechnung gezogen werden. Ich habe aber diese Nachwirkung nur soweit berücksichtigen müssen, dass die Zeit abgewartet wurde, bis sich das Tier wieder mit der Grundration in $\mathrm{N}$-Gleichgewicht gesetzt hatte, d. h. die täglichen $\mathrm{N}$-Ausscheidungen Plus-Minus der N-Zufuhr zu schwanken anfingen.

Wäre eine Stickstoffbilanz am Schlusse der drei Reihen die Hauptsache gewesen, so bätte es wohl wenig Sinn gehabt, die Stickstoffzulagen in von $3 \mathrm{zu} 3$ Tagen steigenden Mengen zu verabfolgen; hier ist doch der Grund leicht ersichtlich: es sollte ein 
Bild des ganzen Verlaufs des N-Umsatzes gegeben werden. Hiermit will ich aber nur erklären, weshalb von mir die Nachwirkungen der N-Zufuhr bei der Diskussion der Versuchsresultate nicht berücksichtiơt wurden. Beabsichtigt man jedoch eine vollstäudige N-Bilanz aufzustellen, so steht dem gewiss nichts entgegen, auch wäre nichts einzuwenden, wenn man alle 12 Tage, in denen Zulagen gegeben wurden, zu je einer Periode zusammenfasst. Nur hat man dann die tatsächlich durch die Analyse ermittelten Zahlen in Rechnung zu stellen.

Ich muss bestreiten, dass Kellner zu der Annabme berechtigt ist, das Tier - ein Fleischfresser - hätte durch die ganzen 54 Tage des Versuchs eine Tendenz gehabt, seinen Stickstoffumsatz in steigendem Masse zu erhöhen, so dass es, wäre die Grundration stetig fortgereicht worden, von einem Stickstoffansatz $\mathrm{zu}$ einem i m mer grösser werdenden Stickstoffverlust fortgeschritten wäre. Hierdurch kommt Kellner zu einer, wie man sagen kann, Belastung besonders der dritten Versuchsreihe, die eine so viel bessere Nachwirkung des Blutalbumins herausrechnen lässt und die Wirkung langsamerer oder schnellerer Resorption des Amids verwischt.

Wesentlich wird ein solches Resultat dadurch ermöglieht, dass bei der gewählten Fütterung überhaupt kein erheblicher N-Ansatz am Schluss der Perioden erfolgen konnte. Wäre das beabsichtigt gewesen, so hätte ich nicht den denkbar schlechtesten Weg dazu eingeschlagen, nämlich einseitig Zulagen verdaulichen Stickstoffs zu geben. Hiernach können kleinste Differenzen, wie sie den unvermeidlichen Fehlergrenzen solcher Versuche nahe stehen, zu einer ungerechtfertigten Bedeutung gelangen.

Die Hündin war durch die Vorfütterungen mit der Grundration jedesmal in N-Gleichgewicht gebracht worden. Ich sebe ganz davon ab, dass namhafte Forscher sich beim Fleischfresser ohne weiteres mit sehr kurzen Vorfütterungsperioden begnügen und die Wirkung der darauf folgenden Ennährung aus dem Vergleich mit dem Stoffwechsel bei der Vorfütterung ableiten. Sicher ist man berechtigt, $\mathrm{N}$-Gleichgewicht anzunehmen, wenn der Harnstickstoff durch mehrere Tage um eine Mittelzahl herumschwankt, keine Tendenz zum Steigen oder Fallen zeigt und das Gleiche bei der täglichen N-Bilanz der Fall ist. In allen drei ersten Perioden mit der Grundration kommen übergreifend und wechselnd die gleichen N-Zahlen im Harn vor und 
in der vierten (Schlussperiode) geht die Hündin - allerdings etwas langsam - zum vollen N-Gleichgewicht zurück.

Aus diesen Gründen und im Hinblick auf alle sonstigen, mit Hunden bei Stoffwechselversuchen gemachten Erfahrungen kann ich also nicht zugeben, dass das Versuchstier gleichsam eine innere Tendenz gehabt hätte, seinen Stickstoffumsatz, wäre die Grundration ohne Zulagen fortgereicht worden, progressiv durch 54 Tage zu steigern.

Stellen wir hiernach die $\mathrm{N}$-Bilanz auf, wobei wir hier einmal von dem $\mathrm{N}$ in Haaren usw. absehen können, so ergibt sich für die 12 Versuchstage folgende Übersicht:

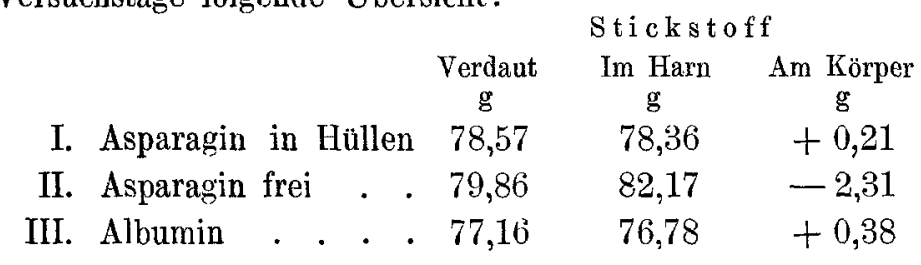

Zuungunsten des "N am Körper" ist noch die Nachwirkung der Perioden zu verrechnen, bis der Harnstickstoff wieder zu den Plus- und Minusschwankungen des N-G̈leichgewichts zurückgeht.

Für Reihe $\mathrm{I} 2$ Tage mit $11,69 \mathrm{~g} \quad-9,98 \mathrm{~g}=1,71 \mathrm{~g} \mathrm{~N}$,

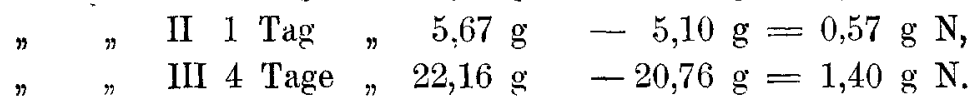

Hiernach bat das Tier in jeder der Reihen infolge starker einseitiger N-Zulagen vom Körper verloren:

Reihe I $=1,50 \mathrm{~g}$, Reihe II $=2,88 \mathrm{~g}$, Reihe III $=1,02 \mathrm{~g}$.

Auch bei dieser Aufstellung kann ich auf die kleine Differenz in Reihe I und III für ganze 12 Tage kein Gewicht legen. Würde man, was wahrscheinich noch richtiger wäre, bei Berechnung der Nachwirkung als mittlere N-Zahl für den täglichen Harn bei Fütterung der Grundration $5,10 \mathrm{~g}$ einsetzen, so würde sich die Differenz noch mehr verringern. Wollte man so geringe Unterschiede überhaupt in Erwägung ziehen, so wäre eben auch zu beachten, dass das zugelegte Eiweiss dem Körper mehr chemisehe Spannkraft zuführt als das Asparagin, dass die etwas geringe $\mathrm{N}$-Aufnahme in der Eiweissperiode weniger steigernd auf den Stoffwechsel wirken müsste u. a. m. Endlich bemerke ich noch, dass die mitgeteilten Zahlen fur das Lebendgewicht des Tieres von mir deswegen nicht diskutiert worden sind, weil aus ihnen bei ihren Schwankungen und schliesslich 
Nochmals zur Wirkung des Asparagins auf den Stickstoffumsatz etc. 451

überhaupt nicht grossen Differenzen keine wesentlichen Folgerungen abgeleitet werden können. Höchstens könnte man aus ihnen in der Reihe mit Verfütterung freien Asparagins eine deutliche Tendenz zum Sinken erkennen.

Um jedem Missverständnis vorzubeugen, möchte ich nicht zu bemerken unterlassen, dass ich beim Fleischfresser Asparagin und Eiweisse, wenn man nur die Resorption des ersteren genügend verlangsamt, gewiss nicht schlechtweg für gleichwertig ansehe; auch nicht, wenn man den Wärmewert der Stoffe etwa durch Zulage von Stärke ausgleichen würde. Bei genügend starken N-Zulagen würden sicher Befunde gemacht werden, die eine Überlegenheit von Eiweiss dartun.

Überhaupt ist die Eiweissreihe in den vorliegenden Versuchen mehr nebensächlich und nur als Vergleichsbild zu den beiden ersten Reihen zu betrachten. Das Wesentliche ist der Nachweis, dass die Resorptionsbedingungen für ein Amid in geschilderter Weise von grossem Einfluss auf den Stoffwechsel sind. Und dieser $\mathrm{Nach}$ weis ist in den Versuchen unbestreitbar geliefert. 